\title{
Revised Mathematical Morphological Concepts
}

\author{
Joseph Ackora-Prah, Yao Elikem Ayekple, Robert Kofi Acquah, Perpetual Saah Andam, \\ Eric Adu Sakyi, Daniel Gyamfi \\ Department of Mathematics, Kwame Nkrumah University of Science and Technology, Kumasi, Ghana \\ Email: ackoraprah@yahoo.co.uk, jegyaoo@gmail.com, racquah@nims.edu.gh, \\ pepandam.andam@yahoo.com, ericadusakyi@ymail.com, quamegyamfi@yahoo.co.uk
}

Received 17 February 2015; accepted 12 March 2015; published 23 March 2015

Copyright (C) 2015 by authors and Scientific Research Publishing Inc.

This work is licensed under the Creative Commons Attribution International License (CC BY). http://creativecommons.org/licenses/by/4.0/

\section{c) (7) Open Access}

\begin{abstract}
We revise some mathematical morphological operators such as Dilation, Erosion, Opening and Closing. We show proofs of our theorems for the above operators when the structural elements are partitioned. Our results show that structural elements can be partitioned before carrying out morphological operations.
\end{abstract}

\section{Keywords}

\section{Mathematical Morphology, Dilation, Erosion, Opening, Closing}

\section{Introduction}

Mathematical morphology is the theory and technique for the analysis and processing of geometrical structures, based on set theory, lattice theory, topology, and random functions. We consider classical mathematical morphology as a field of nonlinear geometric image analysis, developed initially by Matheron [1], Serra [2] and their collaborators, which is applied successfully to geological and biomedical problems of image analysis. The basic morphological operators were developed first for binary images based on set theory [1] [2] inspired by the work of Minkowski [3] and Hadwiger [4]. They were implemented for gray level images based on local min/max operators and level sets [2] [5] or on fuzzy sets [6] [7]. They were also implemented for gray level images with weighted $\mathrm{min} / \mathrm{max}$ operators using a geometric interpretation based on the umbra approach of Sternberg [8] [9] which was algebraically equivalent to maxplus convolutions.

In the development of mathematical morphology in the mid-1960s by Georges Matheron and Jean Serra, they heavily stressed the mathematical formalism on mathematical morphology, and in the work of Haralick, Sternberg and Zhuang [10], the algebraic structure of morphological operators was given. 
In this paper, we outline in details the mathematical morphological operators and their algebraic structures when they are linked with union and intersection. We show that the partitioning of structural element before morphological operations is possible.

\section{Preliminaries}

\subsection{Definitions}

The following definitions are important for our purpose.

\subsubsection{Dilation}

Let the image set $X$ and the structuring element $B$ be subsets of the discrete space $Z^{2}: X \subset Z^{2}, B \subset Z^{2}$. The dilation of $X$ by $B$ is defined as $X \oplus B=\left\{c=x+b \in Z^{2}: x \in X ; b \in B\right\}$; or the Dilation of a binary image $A$ by structure element $B$, is $A \oplus B=\{a+b \mid$ for $a \in A$ and $b \in B\}$.

The dilation transform generally causes image objects to grow in size. From the definitions above, dilation is equivalent to a union of translates of the original image with respect to the structure element, that is, $A \oplus B=\bigcup_{b \in B} A_{b}$.

\subsubsection{Erosion}

Let the image set $X$ and the structuring element $B$ be subsets of the discrete space $Z^{2}: X \subset Z^{2}, B \subset Z^{2}$. The erosion of $X$ by $B$ is defined as $X \ominus B=\left\{c \in Z^{2}: \forall b \in B, \exists x \in X\right.$ where $\left.c=x-b\right\}$; or the Erosion of a binary image $A$ by structure element $B$, is $A \ominus B=\{p \mid p+b \in A \forall b \in B\}$.

Similarly erosion transform allows image objects to shrink in size, that is, $A \ominus B=\bigcap_{b \in B} A_{-b}$.

\subsubsection{Opening}

Let the image set $X$ and the structuring element $B$ be subsets of the discrete space $Z^{2}: X \subset Z^{2}, B \subset Z^{2}$. The Opening of $X$ by $B$ is defined as $X \circ B=(X \ominus B) \oplus B$; or the Opening of a binary image $A$ by structure element $B$, is $A \circ B=(A \odot B) \oplus B$.

\subsubsection{Closing}

Let the image set $X$ and the structuring element $B$ be subsets of the discrete space $Z^{2}: X \subset Z^{2}, B \subset Z^{2}$. The Closing of $X$ by $B$ is defined as $X \bullet B=(X \oplus B) \ominus B$; or the Closing of a binary image $A$ by structure element $B$, is $A \bullet B=(A \oplus B) \oplus B$.

\subsection{Algebraic Properties of Dilation and Erosion}

We note that Dilation is commutative and associative, that is, $A \oplus B=B \oplus A$ and $(A \oplus B) \oplus C=A \oplus(B \oplus C)$, where as Erosion is non-commutative and non-associative, that is, $A \ominus B \neq B \ominus A$ and $(A \odot B) \ominus C \neq A \odot(B \odot C)$ respectively.

Furthermore, Dilation and Erosion are both translation invariant, that is, if $x$ is a vector belonging to $A$ and $B(x \in A, x \in B)$, then $A \oplus B_{x}=A_{x} \oplus B=(A \oplus B)_{x}$ and $A \oplus B_{x}=A_{x} \ominus B=(A \ominus B)_{x}$. Also both Dilation and Erosion are increasing in $A$, that is, if an image set $A_{1}$ is a subset of $A_{2} \quad\left(A_{1} \subset A_{2}\right)$, then $A_{1} \oplus B \subset A_{2} \oplus B$ and $A_{1} \ominus B \subset A_{2} \ominus B$. However, Erosion is decreasing in $B$, that is, if a structuring element $B_{1}$ is a subset of $B_{2}\left(B_{1} \subset B_{2}\right)$, then $A \ominus B_{1} \supset A \ominus B_{2}$. Dilation and Erosion transforms are duals of each other, that is, $(A \oplus B)^{c}=A^{c} \ominus \breve{B}$ and $(A \ominus B)^{c}=A^{c} \oplus \breve{B}$. Dilation and Erosion are also not the inverse of each other, that is, $(A \oplus B) \ominus B \neq A$ and $(A \ominus B) \oplus B \neq A$. Both the dilation and erosion transforms have an identity set, $I$, such 
that $A \oplus I=A$ and $A \oplus I=A$. Dilation transform has an empty set, that is, $\varnothing$ such that $A \oplus \varnothing=\varnothing$.

\subsection{Algebraic Properties of Opening and Closing}

We have Opening and Closing transforms as duals of each other, $(A \circ B)^{c}=A^{c} \bullet \breve{B}$ and $(A \bullet B)^{c}=A^{c} \circ \breve{B}$ but Opening and Closing are not the inverse of each other, $(A \circ B) \bullet B \neq A$ and $(A \bullet B) \circ B \neq A$. Also both Opening and Closing are translation invariant, if $x$ is a vector belonging to $A$ and $B$, then $A \circ B_{x}=A_{x} \circ B=(A \circ B)_{x}$ and $A \bullet B_{x}=A_{x} \bullet B=(A \bullet B)_{x}$. The opening transform is anti-extensive, the Opening of $A$ by a structuring element $B$ is always contained in $A$, regardless of $B \quad(A \circ B \subseteq A)$. The Closing transform is extensive, the Closing of $A$ by a structuring element $B$ always contains $A$, regardless of $B$ $(A \subseteq A \bullet B)$.

Furthermore, Opening and Closing are both increasing in $A$. If an image set $A_{1}$ is a subset of or equal to $A_{2}$ ( $A_{1} \subseteq A_{2}$ ), then $A_{1} \circ B \subseteq A_{2} \circ B$ and $A_{1} \bullet B \subseteq A_{2} \bullet B$ but only Opening is decreasing in $B$, if a structuring element $B_{1}$ is a subset of $B_{2}\left(B_{1} \subset B_{2}\right)$, then $A \circ B_{1} \supset A \circ B_{2}$. Finally, opening and Closing transforms are both idempotent, $A \circ B \circ B=A \circ B$ and $A \bullet B \bullet B=A \bullet B$. However if $X$ is unchanged by opening with $B, X$ is said to be open, whereas if $X$ is unchanged by closing with $B, X$ is said to be closed.

\section{Results}

In this section we present unions and intersections of Dilation, Erosion, Opening and Closing of two different sets and their extensions. The following theorems and their proofs will help us to describe the various results.

\section{The morphological operators with $\boldsymbol{n}$ distinct sets}

\section{Theorem 1 (The union of Dilation with $\boldsymbol{n}$ differents sets)}

If $A=A_{1} \cup A_{2} \cup A_{3} \cup \cdots \cup A_{n}$ for $n \geq 2$

Then $A \oplus B=\left(A_{1} \oplus B\right) \cup\left(A_{2} \oplus B\right) \cup\left(A_{3} \oplus B\right) \cup \cdots \cup\left(A_{n} \oplus B\right)$

Proof.

If $A=A_{1} \cup A_{2}$

Then

$$
\begin{aligned}
\left(A_{1} \oplus B\right) \bigcup\left(A_{2} \oplus B\right) & =\bigcup_{b \in B} A_{1_{b}} \cup \bigcup_{b \in B} A_{2_{b}}=\bigcup_{b \in B}\left\{A_{1_{b}} \cup A_{2_{b}}\right\}=\bigcup_{b \in B}\left(A_{1} \cup A_{2}\right)_{b} \\
& =\left(A_{1} \cup A_{2}\right) \oplus B=A \oplus B
\end{aligned}
$$

This implies $A \oplus B=\left(A_{1} \cup A_{2}\right) \oplus B=\left(A_{1} \oplus B\right) \cup\left(A_{2} \oplus B\right)$

Assume that if $A=A_{1} \cup A_{2} \cup A_{3} \cup \cdots \cup A_{k}$ for $k \geq 2$

Then $A \oplus B=\left(A_{1} \oplus B\right) \cup\left(A_{2} \oplus B\right) \cup\left(A_{3} \oplus B\right) \cup \cdots \cup\left(A_{k} \oplus B\right)$

Now we show that if $A=A_{1} \cup A_{2} \cup A_{3} \cup \cdots \cup A_{k} \cup A_{k+1}$

Then

$$
\begin{aligned}
A \oplus B & =\left(A_{1} \cup A_{2} \cup A_{3} \cup \cdots \cup A_{k} \cup A_{k+1}\right) \oplus B \\
& =\left[\left(A_{1} \cup A_{2} \cup A_{3} \cup \cdots \cup A_{k}\right) \cup A_{k+1}\right] \oplus B \\
& =\left[\left(A_{1} \cup A_{2} \cup A_{3} \cup \cdots \cup A_{k}\right) \oplus B\right] \cup\left(A_{k+1} \oplus B\right) \\
& =\left(A_{1} \oplus B\right) \cup\left(A_{2} \oplus B\right) \cup\left(A_{3} \oplus B\right) \cup \cdots \cup\left(A_{k} \oplus B\right) \cup\left(A_{k+1} \oplus B\right)
\end{aligned}
$$

Theorem 2 (The intersection of Dilation with $\boldsymbol{n}$ different sets)

If $A=A_{1} \cap A_{2} \cap A_{3} \cap \cdots \cap A_{n}$ for $n \geq 2$ 
Then $A \oplus B=\left(A_{1} \oplus B\right) \cap\left(A_{2} \oplus B\right) \cap\left(A_{3} \oplus B\right) \cap \cdots \cap\left(A_{n} \oplus B\right)$

Proof.

If $A=A_{1} \cap A_{2}$

Then

$$
\begin{aligned}
\left(A_{1} \oplus B\right) \cap\left(A_{2} \oplus B\right) & =\bigcup_{b \in B} A_{1_{b}} \cap \bigcup_{b \in B} A_{2_{b}}=\bigcup_{b \in B}\left\{A_{1_{b}} \cap A_{2_{b}}\right\}=\bigcup_{b \in B}\left(A_{1} \cap A_{2}\right)_{b} \\
& =\left(A_{1} \cap A_{2}\right) \oplus B=A \oplus B
\end{aligned}
$$

This implies $A \oplus B=\left(A_{1} \cap A_{2}\right) \oplus B=\left(A_{1} \oplus B\right) \cap\left(A_{2} \oplus B\right)$

Assume that if $A=A_{1} \cap A_{2} \cap A_{3} \cap \cdots \cap A_{k}$ for $k \geq 2$

Then $A \oplus B=\left(A_{1} \oplus B\right) \cap\left(A_{2} \oplus B\right) \cap\left(A_{3} \oplus B\right) \cap \cdots \cap\left(A_{k} \oplus B\right)$

Now we show that if $A=A_{1} \cap A_{2} \cap A_{3} \cap \cdots \cap A_{k} \cap A_{k+1}$

Then

$$
\begin{aligned}
A \oplus B & =\left(A_{1} \cap A_{2} \cap A_{3} \cap \cdots \cap A_{k} \cap A_{k+1}\right) \oplus B \\
& =\left[\left(A_{1} \cap A_{2} \cap A_{3} \cap \cdots \cap A_{k}\right) \cap A_{k+1}\right] \oplus B \\
& =\left[\left(A_{1} \cap A_{2} \cap A_{3} \cap \cdots \cap A_{k}\right) \oplus B\right] \cap\left(A_{k+1} \oplus B\right) \\
& =\left(A_{1} \oplus B\right) \cap\left(A_{2} \oplus B\right) \cap\left(A_{3} \oplus B\right) \cap \cdots \cap\left(A_{k} \oplus B\right) \cap\left(A_{k+1} \oplus B\right)
\end{aligned}
$$

\section{Theorem 3 (The union of Erosion with $\boldsymbol{n}$ distinct sets)}

If $A=A_{1} \cup A_{2} \cup A_{3} \cup \cdots \cup A_{n}$ for $n \geq 2$

Then $A \ominus B=\left(A_{1} \ominus B\right) \cup\left(A_{2} \ominus B\right) \cup\left(A_{3} \ominus B\right) \cup \cdots \cup\left(A_{n} \ominus B\right)$

Proof.

If $A=A_{1} \cup A_{2}$

Then

$$
\begin{aligned}
\left(A_{1} \ominus B\right) \cup\left(A_{2} \ominus B\right) & =\bigcap_{b \in B} A_{1_{-b}} \cup \bigcap_{b \in B} A_{2_{-b}}=\bigcap_{b \in B}\left\{A_{1_{-b}} \cup A_{2_{-b}}\right\}=\bigcap_{b \in B}\left(A_{1} \cup A_{2}\right)_{-b} \\
& =\left(A_{1} \cup A_{2}\right) \ominus B=A \ominus B
\end{aligned}
$$

This implies $A \odot B=\left(A_{1} \cup A_{2}\right) \ominus B=\left(A_{1} \ominus B\right) \cup\left(A_{2} \ominus B\right)$

Assume that if $A=A_{1} \cup A_{2} \cup A_{3} \cup \cdots \cup A_{k}$ for $k \geq 2$

Then $A \ominus B=\left(A_{1} \ominus B\right) \cup\left(A_{2} \ominus B\right) \cup\left(A_{3} \ominus B\right) \cup \cdots \cup\left(A_{k} \ominus B\right)$

Now we show that if $A=A_{1} \cup A_{2} \cup A_{3} \cup \cdots \cup A_{k} \cup A_{k+1}$

Then

$$
\begin{aligned}
A \ominus B & =\left(A_{1} \cup A_{2} \cup A_{3} \cup \cdots \cup A_{k} \cup A_{k+1}\right) \ominus B \\
& =\left[\left(A_{1} \cup A_{2} \cup A_{3} \cup \cdots \cup A_{k}\right) \cup A_{k+1}\right] \ominus B \\
& =\left[\left(A_{1} \cup A_{2} \cup A_{3} \cup \cdots \cup A_{k}\right) \ominus B\right] \cup\left(A_{k+1} \ominus B\right) \\
& =\left(A_{1} \ominus B\right) \cup\left(A_{2} \ominus B\right) \cup\left(A_{3} \ominus B\right) \cup \cdots \cup\left(A_{k} \ominus B\right) \cup\left(A_{k+1} \ominus B\right)
\end{aligned}
$$

\section{Theorem 4 (The intersection of Erosion with $\boldsymbol{n}$ distinct sets)}

If $A=A_{1} \cap A_{2} \cap A_{3} \cap \cdots \cap A_{n}$ for $n \geq 2$

Then $A \ominus B=\left(A_{1} \ominus B\right) \cap\left(A_{2} \ominus B\right) \cap\left(A_{3} \ominus B\right) \cap \cdots \cap\left(A_{n} \ominus B\right)$

Proof. 
If $A=A_{1} \cap A_{2}$

Then

$$
\begin{aligned}
\left(A_{1} \ominus B\right) \cap\left(A_{2} \ominus B\right) & =\bigcap_{b \in B} A_{1_{-b}} \cap \bigcap_{b \in B} A_{2_{-b}}=\bigcap_{b \in B}\left\{A_{1_{-b}} \cap A_{2_{-b}}\right\}=\bigcap_{b \in B}\left(A_{1} \cap A_{2}\right)_{-b} \\
& =\left(A_{1} \cup A_{2}\right) \ominus B=A \ominus B
\end{aligned}
$$

This implies $\left(A_{1} \cap A_{2}\right) \ominus B=\left(A_{1} \ominus B\right) \cap\left(A_{2} \ominus B\right)$

Assume that if $A=A_{1} \cap A_{2} \cap A_{3} \cap \cdots \cap A_{k}$ for $k \geq 2$

Then $A \ominus B=\left(A_{1} \ominus B\right) \cap\left(A_{2} \ominus B\right) \cap\left(A_{3} \ominus B\right) \cap \cdots \cap\left(A_{k} \ominus B\right)$

Now we show that if $A=A_{1} \cap A_{2} \cap A_{3} \cap \cdots \cap A_{k} \cap A_{k+1}$

Then

$$
\begin{aligned}
A \ominus B & =\left(A_{1} \cap A_{2} \cap A_{3} \cap \cdots \cap A_{k} \cap A_{k+1}\right) \ominus B \\
& =\left[\left(A_{1} \cap A_{2} \cap A_{3} \cap \cdots \cap A_{k}\right) \cap A_{k+1}\right] \ominus B \\
& =\left[\left(A_{1} \cap A_{2} \cap A_{3} \cap \cdots \cap A_{k}\right) \ominus B\right] \cap\left(A_{k+1} \ominus B\right) \\
& =\left(A_{1} \ominus B\right) \cap\left(A_{2} \ominus B\right) \cap\left(A_{3} \ominus B\right) \cap \cdots \cap\left(A_{k} \ominus B\right) \cap\left(A_{k+1} \ominus B\right)
\end{aligned}
$$

\section{Theorem 5 (The union of Opening with $\boldsymbol{n}$ different sets)}

If $A=A_{1} \cup A_{2} \cup A_{3} \cup \cdots \cup A_{n}$ for $n \geq 2$

Then $A \circ B=\left(A_{1} \circ B\right) \cup\left(A_{2} \circ B\right) \cup\left(A_{3} \circ B\right) \cup \cdots \cup\left(A_{n} \circ B\right)$

Proof.

If $A=A_{1} \cup A_{2}$

Then

$$
\begin{aligned}
\left(A_{1} \circ B\right) \cup\left(A_{2} \circ B\right) & =\left(A_{1} \ominus B\right) \oplus B \cup\left(A_{2} \ominus B\right) \oplus B=\left[\left(A_{1} \ominus B\right) \cup\left(A_{2} \ominus B\right)\right] \oplus B \\
& =\left[\left(A_{1} \cup A_{2}\right) \ominus B\right] \oplus B=\left(A_{1} \cup A_{2}\right) \circ B=A \circ B
\end{aligned}
$$

This implies $A \circ B=\left(A_{1} \cup A_{2}\right) \circ B=\left(A_{1} \circ B\right) \cup\left(A_{2} \circ B\right)$

Assume that if $A=A_{1} \cup A_{2} \cup A_{3} \cup \cdots \cup A_{k}$ for $k \geq 2$

Then $A \circ B=\left(A_{1} \circ B\right) \cup\left(A_{2} \circ B\right) \cup\left(A_{3} \circ B\right) \cup \cdots \cup\left(A_{k} \circ B\right)$

Now we show that if $A=A_{1} \cup A_{2} \cup A_{3} \cup \cdots \cup A_{k} \cup A_{k+1}$

Then

$$
\begin{aligned}
A \circ B & =\left(A_{1} \cup A_{2} \cup A_{3} \cup \cdots \cup A_{k} \cup A_{k+1}\right) \circ B \\
& =\left[\left(A_{1} \cup A_{2} \cup A_{3} \cup \cdots \cup A_{k}\right) \cup A_{k+1}\right] \circ B \\
& =\left[\left(A_{1} \cup A_{2} \cup A_{3} \cup \cdots \cup A_{k}\right) \circ B\right] \cup\left(A_{k+1} \circ B\right) \\
& =\left(A_{1} \circ B\right) \cup\left(A_{2} \circ B\right) \cup\left(A_{3} \circ B\right) \cup \cdots \cup\left(A_{k} \circ B\right) \cup\left(A_{k+1} \circ B\right)
\end{aligned}
$$

\section{Theorem 6 (The intersection of Opening with $\boldsymbol{n}$ different sets)}

If $A=A_{1} \cap A_{2} \cap A_{3} \cap \cdots \cap A_{n}$ for $n \geq 2$

Then $A \circ B=\left(A_{1} \circ B\right) \cap\left(A_{2} \circ B\right) \cap\left(A_{3} \circ B\right) \cap \cdots \cap\left(A_{n} \circ B\right)$

Proof.

If $A=A_{1} \cap A_{2}$

Then 


$$
\begin{aligned}
\left(A_{1} \circ B\right) \cap\left(A_{2} \circ B\right) & =\left(A_{1} \ominus B\right) \oplus B \cap\left(A_{2} \ominus B\right) \oplus B=\left[\left(A_{1} \ominus B\right) \cap\left(A_{2} \ominus B\right)\right] \oplus B \\
& =\left[\left(A_{1} \cap A_{2}\right) \ominus B\right] \oplus B=\left(A_{1} \cap A_{2}\right) \circ B=A \circ B
\end{aligned}
$$

This implies $A \circ B=\left(A_{1} \cap A_{2}\right) \circ B=\left(A_{1} \circ B\right) \cap\left(A_{2} \circ B\right)$

Assume that if $A=A_{1} \cap A_{2} \cap A_{3} \cap \cdots \cap A_{k}$ for $k \geq 2$

Then $A \circ B=\left(A_{1} \circ B\right) \cap\left(A_{2} \circ B\right) \cap\left(A_{3} \circ B\right) \cap \cdots \cap\left(A_{k} \circ B\right)$

Now we show that if $A=A_{1} \cap A_{2} \cap A_{3} \cap \cdots \cap A_{k} \cap A_{k+1}$

Then

$$
\begin{aligned}
A \circ B & =\left(A_{1} \cap A_{2} \cap A_{3} \cap \cdots \cap A_{k} \cap A_{k+1}\right) \circ B \\
& =\left[\left(A_{1} \cap A_{2} \cap A_{3} \cap \cdots \cap A_{k}\right) \cap A_{k+1}\right] \circ B \\
& =\left[\left(A_{1} \cap A_{2} \cap A_{3} \cap \cdots \cap A_{k}\right) \circ B\right] \cap\left(A_{k+1} \circ B\right) \\
& =\left(A_{1} \circ B\right) \cap\left(A_{2} \circ B\right) \cap\left(A_{3} \circ B\right) \cap \cdots \cap\left(A_{k} \circ B\right) \cap\left(A_{k+1} \circ B\right)
\end{aligned}
$$

\section{Theorem 7 (The union of Closing with $\boldsymbol{n}$ distinct sets)}

If $A=A_{1} \cup A_{2} \cup A_{3} \cup \cdots \cup A_{n}$ for $n \geq 2$

Then $A \bullet B=\left(A_{1} \bullet B\right) \cup\left(A_{2} \bullet B\right) \cup\left(A_{3} \bullet B\right) \cup \cdots \cup\left(A_{n} \bullet B\right)$

Proof.

If $A=A_{1} \cup A_{2}$

Then

$$
\begin{aligned}
\left(A_{1} \bullet B\right) \cup\left(A_{2} \bullet B\right) & \left.=\left(A_{1} \oplus B\right) \ominus B \bigcup\left(A_{2} \oplus B\right) \ominus B=\left[\left(A_{1} \oplus B\right)\right) \cup\left(A_{2} \oplus B\right)\right] \ominus B \\
& =\left[\left(A_{1} \cup A_{2}\right) \oplus B\right] \ominus B=\left(A_{1} \cup A_{2}\right) \bullet B=A \bullet B
\end{aligned}
$$

This implies $A \bullet B=\left(A_{1} \cup A_{2}\right) \bullet B=\left(A_{1} \bullet B\right) \bigcup\left(A_{2} \bullet B\right)$

Assume that if $A=A_{1} \cup A_{2} \cup A_{3} \cup \cdots \cup A_{k}$ for $k \geq 2$

Then $A \bullet B=\left(A_{1} \bullet B\right) \cup\left(A_{2} \bullet B\right) \cup\left(A_{3} \bullet B\right) \cup \cdots \cup\left(A_{k} \bullet B\right)$

Now we show that if $A=A_{1} \cup A_{2} \cup A_{3} \cup \cdots \cup A_{k} \cup A_{k+1}$

Then

$$
\begin{aligned}
A \bullet B & =\left(A_{1} \cup A_{2} \cup A_{3} \cup \cdots \cup A_{k} \cup A_{k+1}\right) \bullet B \\
& =\left[\left(A_{1} \cup A_{2} \cup A_{3} \cup \cdots \cup A_{k}\right) \cup A_{k+1}\right] \bullet B \\
& =\left[\left(A_{1} \cup A_{2} \cup A_{3} \cup \cdots \cup A_{k}\right) \bullet B\right] \cup\left(A_{k+1} \bullet B\right) \\
& =\left(A_{1} \bullet B\right) \cup\left(A_{2} \bullet B\right) \cup\left(A_{3} \bullet B\right) \cup \cdots \cup\left(A_{k} \bullet B\right) \cup\left(A_{k+1} \bullet B\right)
\end{aligned}
$$

\section{Theorem 8 (The intersection of Closing with $\boldsymbol{n}$ distinct sets)}

If $A=A_{1} \cap A_{2} \cap A_{3} \cap \cdots \cap A_{n}$ for $n \geq 2$

Then $A \bullet B=\left(A_{1} \bullet B\right) \cap\left(A_{2} \bullet B\right) \cap\left(A_{3} \bullet B\right) \cap \cdots \cap\left(A_{n} \bullet B\right)$

Proof.

If $A=A_{1} \cap A_{2}$

Then

$$
\begin{aligned}
\left(A_{1} \bullet B\right) \cap\left(A_{2} \bullet B\right) & =\left(A_{1} \oplus B\right) \oplus B \cap\left(A_{2} \oplus B\right) \ominus B=\left[\left(A_{1} \oplus B\right) \cap\left(A_{2} \oplus B\right)\right] \ominus B \\
& =\left[\left(A_{1} \cap A_{2}\right) \oplus B\right] \ominus B=\left(A_{1} \cap A_{2}\right) \bullet B=A \bullet B
\end{aligned}
$$


This implies $A \bullet B=\left(A_{1} \cap A_{2}\right) \bullet B=\left(A_{1} \bullet B\right) \cap\left(A_{2} \bullet B\right)$

Assume that if $A=A_{1} \cap A_{2} \cap A_{3} \cap \cdots \cap A_{k}$ for $k \geq 2$

Then $A \bullet B=\left(A_{1} \bullet B\right) \cap\left(A_{2} \bullet B\right) \cap\left(A_{3} \bullet B\right) \cap \cdots \cap\left(A_{k} \bullet B\right)$

Now we show that if $A=A_{1} \cap A_{2} \cap A_{3} \cap \cdots \cap A_{k} \cap A_{k+1}$

Then

$$
\begin{aligned}
A \bullet B & =\left(A_{1} \cap A_{2} \cap A_{3} \cap \cdots \cap A_{k} \cap A_{k+1}\right) \bullet B \\
& =\left[\left(A_{1} \cap A_{2} \cap A_{3} \cap \cdots \cap A_{k}\right) \cap A_{k+1}\right] \bullet B \\
& =\left[\left(A_{1} \cap A_{2} \cap A_{3} \cap \cdots \cap A_{k}\right) \bullet B\right] \cap\left(A_{k+1} \bullet B\right) \\
& =\left(A_{1} \bullet B\right) \cap\left(A_{2} \bullet B\right) \cap\left(A_{3} \bullet B\right) \cap \cdots \cap\left(A_{k} \bullet B\right) \cap\left(A_{k+1} \bullet B\right)
\end{aligned}
$$

\section{Conclusion}

We have shown that Dilation, Erosion, Opening and Closing of two or more sets with the same structural element and carrying out the union of the outcome is the same as taking the union of the two or more sets and operating the results with the structural element. The above result also holds for the intersection. These operators show ways of partitioning the structural element in order to carry out the morphological operation with ease. Furthermore, the results above give a simplification of morphological operations when dealing with lots of sets with the same structural element.

\section{Acknowledgements}

We are grateful to the Almighty God and the Department of Mathematics, Kwame Nkrumah University of Science and Technology for providing us resources to help complete this research successfully.

\section{References}

[1] Matheron, G. (1975) Random Sets and Integral Geometry. Wiley, New York.

[2] Serra, J.C. (1982) Image Analysis and Mathematical Morphology. Academic Press, New York.

[3] Minkowski, H. (1903) Vorlumen und Oberflache. Mathematische Annalen, 57, 447-495. http://dx.doi.org/10.1007/BF01445180

[4] Hadwiger, H. (1957) Vorlesungen uber Inhalt, Oberflache und isoperimetrie. Springer Verlag, Berlin. http://dx.doi.org/10.1007/978-3-642-94702-5

[5] Meyer, F. (1977) Contrast Feature Extraction. In: Special Issues of Practical Metallography, Ederer Verlag GmbH, Stuttgart, Proc. 2nd European Symp. on Quant. Anal. of Microstruct. in Materials Science, Biology and Medicine, France.

[6] Nakagawa, Y. and Rosenfeld, A. (1978) A Note on the Use of Local Min and Max Operations in Digital Picture Processing. IEEE Transactions on Systems, Man, and Cybernetics, 8, 632-635. http://dx.doi.org/10.1109/TSMC.1978.4310040

[7] Goetcherian, V. (1980) From Binary To Greytone Image Processing Using Fuzzy Logic Concepts. Pattern Recognition, 12, 7-15. http://dx.doi.org/10.1016/0031-3203(80)90049-7

[8] Sternberg, S.R. (1980) Language and Architecture for Parallel Image Processing. In: Gelsema, E. and Kanal, L., Eds., Pattern Recognition in Practice, North Holland Publishing, Holland.

[9] Sternberg, S.R. (1986) Grayscale Morphology. Computer Vision, Graphics and Image Processing, 35, 333-355. http://dx.doi.org/10.1016/0734-189X(86)90004-6

[10] Haralick, R.M., Sternberg, S.R. and Zhuang, X. (1987) Image Analysis Using Mathematical Morphology. IEEE Transactions on Pattern Analysis and Machine Intelligence, 9, 532-550. http://dx.doi.org/10.1109/TPAMI.1987.4767941 